\title{
Adrenaline Sensing by 2D-SPR Observation of the Stem Cell-Induced Cardiac Cell Response
}

\author{
Shiori Kawashima $^{1}$, Hiroaki Shinohara ${ }^{2}$, Minoru Suga ${ }^{2}$ \\ 1 Graduate School of Science and Engineering for Education, University of Toyama, \\ 2 Graduate School of Science and Engineering for Research, University of Toyama, \\ marker0723.kwsm@gmail.com
}

\begin{abstract}
The 2D-SPR system was applied to observe intracellular reactions of the cardiomyocyte differentiated from model stem cell upon the stimulation with various concentration of adrenaline. As expected, adrenaline response in cardiac differentiated cell regions increased in the concentratior range of 1-10 $\mu \mathrm{M}$. The increase of reflected light intensity at 28 minutes after adrenaline stimulation was concentration dependent. Furthermore, we tried to monitor the closstalk of intracellular signal passways via $\alpha$ - and $\beta$ - adrenoceptors which were expressed during cardiac differentiation. It was demonstrated that 2DSPR observeation of the cardiomyocyte response upon adrenaline stimulation was easy and useful for sensing of cardiac differentiated cells. And, we propose that this sensing system may be effective for consideration of the crosstalk in some intracellular signal transduction passways.
\end{abstract}

Key words: 2D-SPR, adrenaline sensing, cardiac differentiation, P19CL6, crosstalk

\section{Background}

The 2D-SPR system can obtain the images which show local refractive index change on the surface of a thin gold film.In our laboratory, we have already succeeded to observe the refractive index change in living upon drug stimulation with 2D-SPR system.we considered those refractive index change may be attibuted to PKC translocation to the bottom cell membrane. [1] In our previous report, the 2DSPR observation was applicable for evaluation of cardiac differentiation of a model stem cell, P19CL6 by muscarine stimulation.P19CL6 cells were differentiated in the presence of $1 \%$ dimethylsulfoxide(DMSO). [2] Here, we focused on the adrenergic receptors which were expressed during myocardial differentiation. And we have tried the evaluation of cardiac differentiation by 2D-SPR observation upon adrenaline stimulation. We reported here the cell-based 2D-SPR sensing to adrenaline and the observation of the crosstalk of the signal passways through $\alpha$ - and $\beta$-adrenoceptors .

\section{Material and Methods}

To induce cardiac differentiation, $1.8 \times 10^{5}$ P19CL6 cells were cultured in a 6-well plate with a-MEM supplemented with $1 \%$ DMSO at $37^{\circ} \mathrm{C}$. The medium was changed every 2 days. After 10days from DMSO treatment differentiation of the cells was confirmed by fluorescent immuno staining with FITC-anticTnT. Undifferentiated(day0) and differentiated (day10) cells were cultured on an Au chip with a silicon chamber for one day, and used to the 2D-SPR observation. Medium was exchanged to Hank's solution (HBSS) for 2D-SPR observation. The SPR imaging was performed at the constant measurement angle with a NTT-AT (Japan)made 2D-SPR equipment. The measurement angle was initially determined from the SPR curves of the cell regions. 


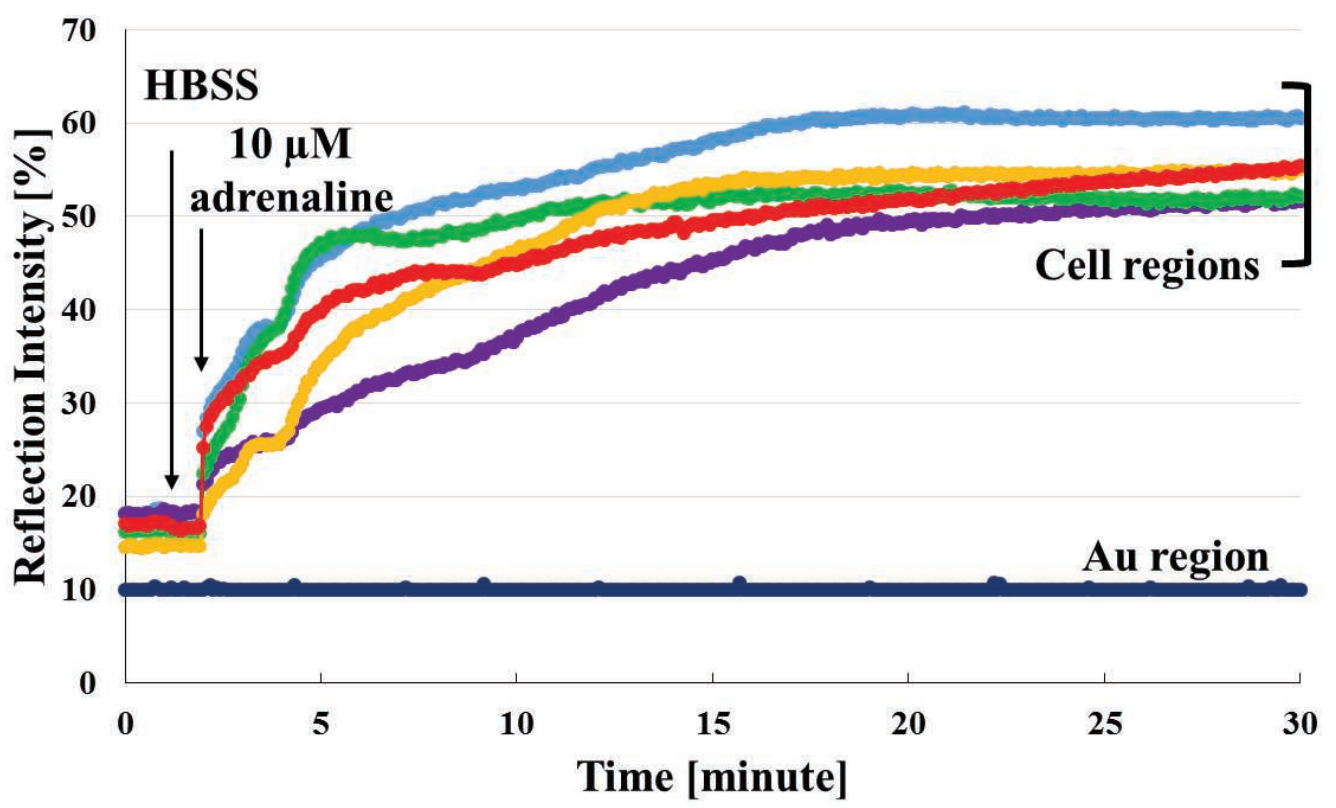

\section{Result and Discussion}

Fig.1 shows the time-course of reflection intensity changes of differentiated (day10) cell region and $\mathrm{Au}$ region upon $10 \mu \mathrm{M}$ adrenaline stimulation. Initially, differentiated cells were stimulated with only HBSS and no changes in refection intensity at cell and Au regions were confirmed. After 1 minute from HBSS injection, differentiated cells were stimulated with $10 \mu \mathrm{M}$ adrenaline and SPR response was observed for 28 minutes. As shown in Fig.1, three steps of rapid increases of reflection intensity were observed for 28 minutes from adrenaline stimulation at cell regions. The reflection intensity changes were measured after 28 minutes from the stimulation with various concentration of adrenaline $(0,1,5,10 \mu \mathrm{M})$. This cell-based sensor could detect adrenaline in dose-dependent manner with the cardiac differentiated cells (day10).

We next considered the closstalk of the signal passways through $\alpha-$ and $\beta$ adrenoceptors, because it is well known that $\alpha$ and $\beta$ - adrenoceptor were expressed during cardiac differentiation. The SPR response upon adrenaline stimulation including

the closstalk signaling was observed in the presence of a few inhibitors to consider what intracellular reactions were monitored by 2DSPR.

These results demonstrated that 2D-SPR observation might be effective in adrenaline sensing and identification of the cardiac differentiated cells and consideration on the closstalk of intracellur signaling.
Fig.1 Time-course of the reflection intensity changes at individual differentiated cell (day10) regions upon $10 \mu \mathrm{M}$ adrenaline stimulation with the 2D-SPR imager.

\section{References}

[1] T. A. Mir, H.Shinohara, Anal.

Biochem., 443(1), 46-51(2013).

[2] S. Kawashima, H. Shinohara et al., Abstract of the ACCS2017. 